\title{
Trastornos alimentarios maternos y su influencia en la conducta alimentaria de sus hijas(os)
}

\author{
R. Behar • M. Arancibia
}

\begin{abstract}
Maternal eating disorders and their influence on eating behavior of the children: a review of the literature
\end{abstract}

Background: There is conclusive evidence about the influence of mothers with eating disorders (ED) on their children. Objective: To describe different aspects about mothers with ED and its implications in the feeding, growing and development in their children. Method: A review of specialized textbooks and the available literature in PubMed was made. Results: Mothers with previous or present ED show restrictive eating patterns, a deficient affective bond with their own mothers, exhibit more psychiatric comorbidities, mainly depression and anxiety; in addition, feelings of guilt and shame, early cessation of breastfeeding, high control of food consumption of their children, and/or pressure them to eat, irregular mealtimes, unbalanced and monotonous meals, proneness to slimness, a critical attitude about the body shape of their daughters. Breastfeeding implies a critical period for the irruption or relapse of an ED due to the adjustment to the characteristic body shape changes during pregnancy. Conclusions: There is a particular relationship between a maternal ED and its occurrence in their children, and it can be considered a risk factor for its development.

(Key words: Eating disorders, children of mothers with eating disorders, anorexia nervosa, bulimia nervosa, pregnancy, breastfeeding).

Rev Chil Pediatr 2014; 85 (6): 731-739

\section{RESUMEN}

Antecedentes: Existe concluyente evidencia de la influencia de madres con trastornos de la conducta alimentaria (TCA) en sus hijas (os). Objetivo: Analizar descriptivamente los diversos aspectos de madres con historia de TCA y sus implicancias en la nutrición, crecimiento y desarrollo de sus hijas (os). Método: Se efectuó una revisión de la literatura disponible en PubMed y textos de consulta especializados. Resultados: Las madres con TCA previo o actual muestran patrones alimentarios restrictivos, un vínculo afectivo deficitario con sus propias madres, exhiben más comorbilidades psiquiátricas, principalmente depresión y ansiedad; además

Recibido el 14 de septiembre de 2013. Última versión aceptada el 14 de septiembre de 2014.

Rosa Behar A. $(\bowtie)$

Médico Psiquiatra, Profesora Titular, Departamento de Psiquiatría, Universidad de Valparaíso.

E-mail: rositabehar@vtr.net

Marcelo Arancibia M.

Alumno de la Escuela de Medicina, Universidad de Valparaíso. 
sentimientos de culpa y vergüenza, cese precoz de la lactancia, elevado control del consumo de alimentos de sus hijas (os) y/o presión para comer, horarios irregulares de comidas, dieta desequilibrada y monótona, preferencia por la delgadez y actitud crítica sobre la silueta corporal de sus hijas (os). La lactancia representa un período crítico para la irrupción o recaída de un TCA por la adaptación que implican los cambios característicos de su figura corporal durante el embarazo. Conclusiones: Existe una particular relación entre la presencia de un TCA materno y su ocurrencia en hijas (os), considerándose un factor de riesgo para su desarrollo.

(Palabras clave: Trastornos de la conducta alimentaria, hijos de madres con trastornos de la conducta alimentaria, anorexia nerviosa, bulimia nerviosa, embarazo, lactancia).

Rev Chil Pediatr 2014; 85 (6): 731-739

\section{Introducción}

La evidencia disponible sugiere que las hijas(os) de madres con patologías alimentarias están en alto riesgo de desarrollar desórdenes en una variedad de ámbitos, incluyendo los trastornos de la conducta alimentaria (TCA). Esta amenaza depende de un amplio rango de factores, siendo frecuentes las alteraciones nutricionales, del crecimiento y desarrollo observadas en las hijas(os) de madres con TCA ${ }^{1}$. Estos desórdenes, etiopatogénicamente multifactoriales, involucran un importante aporte ambiental. En efecto, según Cordella y cols. ${ }^{2}$, los progenitores, mediante el establecimiento de patrones de apego inseguro y escasa elaboración psíquica conjunta de los eventos emocionales, podrían transmitir a sus hijas(os) valores, preocupaciones y conductas relacionadas con la perspectiva sobre la imagen corporal y el peso, pudiendo constituirse este hecho como factor de riesgo para la aparición de un TCA.

En este trabajo se analizarán descriptivamente las dimensiones psicopatológicas y conductuales de las madres con TCA que influyen en los hábitos alimentarios de sus hijas (os), y a su vez, el impacto que este fenómeno supone en el crecimiento y desarrollo de ellas(os).

\section{Metodología}

Se llevó a cabo una investigación de la literatura disponible, a través de la base de datos PubMed y textos de consulta especializados, abarcando desde el año 1987 hasta el 2012, utilizando para la búsqueda palabras claves: eating disorders, anorexia nervosa, bulimia nervosa, mothers, pregnancy y breastfeeding. Se incluyeron, según los criterios de los DSMIII-R ${ }^{3}$ y IV-TR ${ }^{4}$ tanto los TCA completos (anorexia nerviosa restrictiva y compulsivo-purgativa y bulimia nerviosa purgativa) como los parciales (TCA no especificados, ej. trastorno por atracones). Se realizó un análisis descriptivo de la evidencia respecto a diversos aspectos de madres con historia de TCA, tales como hábitos alimentarios, psicopatología comórbida, percepciones y actitudes acerca de la imagen corporal y efectos en el período post parto. Se presenta un caso clínico ilustrativo, previo consentimiento informado de la paciente.

\section{Resultados}

Nuestros hallazgos, relacionados a la impronta en las conductas alimentarias de las hijas(os) de madres con historiales de TCA, arrojaron información de relieve significativo en parámetros tales como conductas alimentarias, comorbilidades psiquiátricas, sentimientos de culpa y vergüenza, vínculo madre/hija(o) y lactancia.

Los TCA constituyen una preocupación para los clínicos abocados a la atención ginecoobstétrica y pediátrica, ya que las gestantes en riesgo de padecerlos pueden adoptar una imagen corporal negativa que persiste en el período postnatal con el aumento de peso adquirido durante el embarazo. Estos cambios físicos pueden reactivar conductas como la restricción alimentaria, atracones o inducción de vómitos para mantener el peso previo al embarazo 5 . 
La literatura señala una asociación específica entre los TCA infantiles y maternos. En niños con anorexia nerviosa, trastorno emocional evitativo alimentario o comer selectivo, 17\% de las madres informó una historia de TCA versus $3 \%$ a $5 \%$ en muestras comunitarias ${ }^{6}$. Stein y cols. ${ }^{7}$, constataron que la alteración del comer en niños de 10 años, se asoció con la duración de la exposición al TCA de la madre. Las niñas(os) de madres con TCA manifestaron hábitos y actitudes alimentarias alteradas en comparación a los controles, pudiendo estar en alto riesgo de desarrollar un franco TCA. Field y cols. ${ }^{8}$, demostraron que niñas menores de 14 años cuyas madres poseían una historia de TCA, exhibían casi 3 veces más posibilidades que sus pares de purgar semanalmente.

\section{Conductas alimentarias}

Farrow y Blissett ${ }^{9}$ y Hoffman y cols. ${ }^{10}$, informaron que las madres con historia de TCA poseían comportamientos restrictivos hacia la alimentación. Otra investigación noruega ${ }^{11}$ ratificó que las madres con bulimia nerviosa y trastorno por atracones, revelaron más estilos de alimentación restrictivos y niños con problemas alimentarios que las madres sin TCA. Según Powell y cols. ${ }^{12}$, estas conductas maternas se asocian con un temperamento infantil sensible, altos niveles de control maternal sobre la alimentación y bajo estímulo para una dieta balanceada y variada, utilizando el alimento para la regulación conductual. Se ha observado que las madres que informaban más rabia y/o frustración durante la alimentación, con más probabilidad presionaban a sus hijos para comer, mientras que las más demandantes restringían más la alimentación, también los presionaban a comer y controlaban su ingesta lipídica ${ }^{13}$. La influencia de los patrones alimentarios maternos potencialmente modificables, sugiere que las interacciones alimentarias entre padres e hijos deberían ser blancos de prevención e intervención durante la infancia temprana.

\section{Comorbilidades}

La co-ocurrencia de trastornos psicopatológicos no sólo es agravante en el caso de presentarse en la hija(o), sino que también representa un factor preponderante de riesgo para el desarrollo de un TCA infantil al evidenciarse en la madre.

García de Amusquibar y de Simone ${ }^{11}$, observaron que las madres de las pacientes con TCA presentaron más episodios de atracones, depresión, abortos provocados y un pobre vínculo afectivo con sus propias madres. Otro análisis indicó que la incidencia de bulimia nerviosa en el primer trimestre del embarazo se asoció significativamente con síntomas de ansiedad, depresión, baja autoestima e insatisfacción con la vida, mientras que la remisión del TCA se correlacionó con autoestima más alta y bienestar vital ${ }^{14}$.

Ammaniti y cols. ${ }^{15}$, confirmaron una asociación significativa entre las características sintomáticas específicas de las madres (actitudes alimentarias alteradas, ansiedad, depresión, hostilidad), de sus hijos (ansiedad/depresión, quejas somáticas y conducta agresiva) y sus modos relacionales disfuncionales durante la alimentación. En niños con problemas alimentarios se han distinguido dos variables significativas del ambiente familiar: desorganización en los horarios de comida y fuerte control materno, mediadores de la asociación entre el TCA infantil y maternal ${ }^{16}$. Asimismo, niveles maternos más altos de comportamientos y preocupaciones en torno a su peso, se asociaron con mayor insatisfacción corporal y prevalencia de conductas para adelgazar en muchachas adolescentes ${ }^{17}$.

\section{Percepción distorsionada del peso y la imagen corporal}

La auto-percepción corporal distorsionada, si bien es parte de la sintomatología clínica de un TCA, no es específica de él, pues no implica necesariamente su diagnóstico ni tampoco configura un sinónimo de tal. Cooley y cols. ${ }^{18}$, incluyeron entre las variables predictoras de la auto-percepción desfavorable de la imagen corporal de sus hijas, la retroalimentación negativa de la madre y la desaprobación de su figura. Además, la tendencia de las madres a internalizar los mensajes de los medios en relación a la delgadez y belleza fue significativa para la predicción y probabilidad de tener hijas con TCA.

En la infancia temprana, la referencia de 
figuras significativas es gravitante en la adquisición de hábitos por parte de las niñas(os), y, en sentido último, en la configuración de su identidad y autoestima. Al respecto, McCabe y cols. ${ }^{19}$, señalan que las actitudes reflejadas por madres y profesoras sobre la apariencia, la dieta y el ejercicio en pre-escolares, comunicaban a sus hijas mensajes dirigidos a perder peso, y a sus hijos a aumentar sus músculos, fomentando la insatisfacción por su imagen corporal. Los autores sugieren la aplicación de programas para las madres, profesoras y escolares con el fin de prevenir el desarrollo de TCA en esta población.

Una investigación que correlacionó alteraciones en el comer entre preadolescentes de ambos sexos y variables maternas, arrojó que la mayoría de las madres prefirió cuerpos más delgados para sus hijas(os). La autoestima, el índice de masa corporal materno y las conductas alimentarias predijeron la aparición de un TCA en sus hijas ${ }^{20}$. Otro análisis mostró que la apreciación de las madres sobre el peso de sus hijas predijo conductas restrictivas en las niñas, siendo la percepción de la imagen corporal y los hábitos alimentarios predictores significativos de mayor insatisfacción corporal ${ }^{21}$, una de las manifestaciones nucleares de los TCA ${ }^{22}$.

\section{Sentimientos de culpa y vergüenza}

Si bien las madres que exhiben un TCA latente o clínico pueden proyectar sus aprensiones sobre la imagen corporal a sus hijas(os), al mismo tiempo pueden experimentar sentimientos de culpa y vergüenza, que aunque ocultos, pueden ser intensos y profundos, debiendo considerarse invariablemente en la prevención de la salud mental de la mujer ${ }^{23}$. Según Rortveit y cols. ${ }^{24}$, estos sentimientos de culpa se relacionan con el deseo de ser una madre lo suficientemente buena y la preocupación por no involucrar a los hijos en sus dificultades alimentarias.

\section{Vínculo madre/hija (o)}

De acuerdo a Bowlby ${ }^{25}$, el apego, vínculo emocional que establece la (el) niña (o) con sus padres, le proporciona la seguridad indispensable para un buen desarrollo de su perso- nalidad. En los TCA, el apego con las madres suele ser ambivalente, del tipo evitativo en la anorexia nerviosa, suscitando suspicacia, escepticismo, retraimiento, dificultad para confiar y depender de los demás, mientras que en la bulimia nerviosa predomina el tipo ansioso, generador de relaciones interpersonales inseguras, desconfianza interpersonal y reticencia al compromiso, con gran preocupación por el posible abandono $^{26}$. De hecho, Stein y cols. ${ }^{27}$, enfatizan la importancia que estriba el TCA materno en el desarrollo del mundo interno infantil.

Elfhag y Linné ${ }^{28}$, distinguieron que el TCA $y$ el comer en respuesta a emociones negativas eran más bien compartidos por madres e hijas, que por madres e hijos. Las madres más jóvenes coincidieron con sus hijas en un mayor interés hacia el cuerpo, mientras que las de mayor edad concordaron en conductas alimentarias más restrictivas. Los vínculos madrehija pueden deberse a una transmisión familiar psicológica y genética genérica específica, y a influencias ambientales ligadas al género. Los comportamientos alimentarios de los hijos parecen ser más independientes. En otra exploración, los niños que presentaron detención en su crecimiento y bajo peso para su edad poseían más probablemente madres con historia de anorexia nerviosa, siendo alimentados insuficientemente, con deprivación psicológica, además de presentar una secreción disminuida de hormona del crecimiento $^{29}$. Asimismo, Russell y cols. ${ }^{30}$, detectaron que hijas(os) de madres anorécticas habían sufrido deprivación alimentaria con severa reducción del peso para la edad y la talla, transmitiendo estas madres sus preocupaciones patológicas por el tamaño corporal a sus hijas(os). La psicoterapia familiar combinada con hospitalizaciones facilitó la normalización del crecimiento en sus hijas(os), por lo que es esencial obtener la confianza de las madres anorécticas sospechosas de mal nutrirlas(os) y adoptar una aproximación terapéutica hacia la familia completa. Por otra parte, se ha constatado que las madres de hijas con TCA se encontraban más insatisfechas con el funcionamiento del sistema familiar, presentaban más TCA y pensaban que sus hijas debían perder más peso comparadas con las madres de las niñas sin $\mathrm{TCA}^{31}$. 


\section{Lactancia}

Ha sido demostrado que un amamantamiento prolongado favorece un apego vincular positivo madre-hija(o), de suma importancia como ya fue expuesto, ayuda a la pérdida de peso postparto y proporciona a la madre una oportunidad para restablecer hábitos alimentarios saludables para su hija(o) ${ }^{32}$.

Un estudio noruego ${ }^{33}$ comparó la prevalencia de lactancia en mujeres con anorexia nerviosa, bulimia nerviosa, trastorno por atracones y TCA no especificado, con mujeres sin TCA durante los primeros 6 meses después del nacimiento. Casi todas las mujeres (98\%) inicialmente amamantaron a sus hijos, sin diferencia estadísticamente significativa entre los subgrupos con y sin TCA. Sin embargo, el riesgo de cese temprano antes de los 6 meses postparto, aumentó para todos los subgrupos de madres con TCA. Respecto a la adaptación al índice de masa corporal, edad, educación, peso de nacimiento y parto pre-término, sólo las madres con anorexia nerviosa y TCA no especificado habían aumentado el riesgo de cese de amamantamiento. Estos resultados refuerzan aun más el concepto de que algunos TCA pueden influir las prácticas alimentarias tempranas de las madres y que es menester el apoyo adicional para ayudar a las mujeres con anorexia nerviosa a mantener la lactancia debido a la importancia que en ella radica.

La conducta alimentaria en mujeres con TCA aparece como problemática para el embarazo, manifestándose consecuentemente desde bajo peso al nacer, hasta dificultades en la alimentación por pecho, distanciamiento afectivo $\mathrm{y}$ ausencia de interacción en los horarios de comidas. Aunque estos factores probablemente no causen los TCA, pueden contribuir a un ambiente facilitador en el cual la predisposición genética se exprese con mayor probabilidad. Waugh y Bulik ${ }^{34}$ señalaron que las madres con TCA tuvieron más dificultad en mantener el amamantamiento y expresaron significativamente más comentarios negativos sobre los alimentos y el comer que las madres control durante los horarios de comida.

Agras y cols. ${ }^{35}$, comprobaron que las hijas de madres con TCA eran alimentadas en un horario menos regular, el alimento era usado para propósitos no nutritivos, fueron destetadas más tardíamente, demostraron afectos más negativos y alta avidez por alimentarse tempranamente en la vida. Las madres evidenciaron una preocupación significativamente mayor sobre el peso de sus hijas que aquéllas sin TCA. En efecto, en un estudio cualitativo de mujeres puérperas ${ }^{36}$ con un TCA pre-existente, para perder el peso ganado durante el embarazo, éstas mostraron un temprano regreso al comer restrictivo, drásticos regímenes de ejercicio y/o conductas de atracones y/o purgas.

\section{Comentario}

La evidencia apunta hacia una particular relación entre la ocurrencia de TCA en madres, ya sea como antecedente de vida y/o desorden actual, y la existencia de TCA en sus hijas (os), siendo por lo tanto un factor de riesgo para desarrollarlo. Generalmente las madres con historia de TCA persisten en exhibir hábitos alimentarios restrictivos, presentan un vínculo afectivo deficitario con sus propias madres, desarrollan más comorbilidades psiquiátricas, tales como depresión y ansiedad, y en algunas se observan profundos sentimientos de culpa y vergüenza por no cumplir satisfactoriamente su rol materno e inquietud por no interferir en la nutrición de sus hijas(os), debido a sus problemas alimentarios, los que habitualmente se evidencian mediante la tendencia al cese precoz de la lactancia, gran control de la ingesta de sus hijas(os), en ocasiones presión para comer, horarios irregulares de comidas, énfasis en nutrientes no procesados y orgánicos ${ }^{10}$, dieta desequilibrada y poco variada, preferencia por la delgadez y actitud crítica y descalificadora de la figura corporal de sus hijas $(\mathrm{os})^{22}$, tal como se expone en el caso clínico descrito (tabla 1), en donde se muestra a una paciente significativamente interferida en su funcionamiento cotidiano, particularmente en su actual rol materno. A su vez, ella vivenció la influencia de su propia madre, con mensajes confundentes enfocados hacia la construcción de una corporalidad alterada relativa a aspectos alimentarios, ponderales y de su silueta y que inexorablemente y de manera similar re- 
Paciente de 25 años, primípara, puérpera de 8 semanas, con antecedente de anorexia nerviosa restrictiva tratada a los 14 años, refiere sentirse muy desolada al mirarse en el espejo, por encontrarse con su cuerpo "deformado y terriblemente gordo, parezco una vaca", ya que subió 15 kilos durante su embarazo, a pesar de haber cuidado meticulosamente su dieta. Comenzó hace 6 semanas a restringir drásticamente los hidratos de carbono; no obstante, se induce el vómito, hasta 2 veces al día, especialmente cuando presenta atracones vespertinos de chocolates, helados y pasteles, con lo que ha logrado bajar 5 kilos. Además manifiesta estar más sensible, llora por todo, se desvela en las noches, está irritable con su esposo y el bebé, le molesta cuando llora y lo rechaza en los momentos en que debe amamantarlo. Con mucha culpa y angustia, se arrepiente de haber dado a luz a su hijo, teme que su lactancia se interrumpa, pero ha descuidado los horarios de alimentación porque duerme desordenadamente durante el día. Le ha solicitado a su madre que se haga cargo de rellenos y cuando ella los prepara, mide minuciosamente las cantidades y prefiere los alimentos hipocalóricos y orgánicos. Igualmente su madre sufrió problemas alimentarios en su juventud, y la paciente recuerda que siempre estaba preocupada de su figura corporal: se efectuó una liposucción, continuamente hacía dietas y ejercicio físico, obligándola a practicarlos también a ella. Además, constantemente le criticaba "los rollitos" y la cantidad de alimentos que ingería, pero a la vez, la premiaba con dulces cuando obtenía buenas notas. En nuestra paciente, el psiquiatra, de común acuerdo con el pediatra, indicó suspender la lactancia, además de prescribir fluoxetina, clonazepam y psicoterapia cognitivo-conductual, medidas con las que ha logrado una disminución significativa de su sintomatología depresivo-angustiosa y de sus conductas alimentarias alteradas.

percutirá en mayor o menor grado en la salud física y mental de su hijo. Así como lo señalado anteriormente por Koubaa y cols. ${ }^{5}$, en la paciente del caso es factible de constatar una reactivación de su antiguo TCA, emergiendo posiblemente producto de la interacción entre distorsión de la imagen corporal latente y persistente con su estado puerperal y todos los cambios físicos que él conlleva. Además, exhibe conductas ambivalentes frente a la lactancia, ya que si bien por un lado dice estar preocupada por la interrupción de la lactancia, por otro rechaza el contacto con su hijo a la hora de amamantarlo, delegando esta responsabilidad en su madre. Esta actitud de lejanía afectiva con su hijo va en directo detrimento de la configuración de un apego seguro, con riesgo de constituirse el vínculo materno-infantil en uno de carácter evitativo o ansioso, con todas las consecuencias negativas ya mencionadas.

Los padres son fuertes comunicadores de las presiones socioculturales. Se ha comprobado que sus influencias verbales y estímulo activo poseen gran impacto en las preocupaciones corporales de sus hijas(os) y en sus conductas alimentarias ${ }^{37}$. El análisis de las características individuales de la niña (o), de la madre y de su relación durante el desarrollo de los patrones alimentarios en los primeros 3 años de vida, es extremadamente importante en la evaluación clínica de los TCA tempranos. Blissett y Haycraft $^{38}$ establecieron que las prácticas alimentarias controladas se relacionan con síntomas de TCA en grupos no clínicos de padres y madres, destacando que la preocupación y la dificultad respecto al comer pueden ser transmitidas dentro de las familias. No obstante, Cordella y cols. ${ }^{2}$, no detectaron diferencias significativas al comparar los puntajes de las subescalas del Inventario de Desórdenes Alimentarios- $2^{39}$ al evaluar díadas madres e hijas(os) adolescentes con y sin TCA, infiriendo que no hubo diferencias relevantes entre las conductas, actitudes y creencias relacionadas al alimento.

Se ha demostrado que la ocurrencia de un TCA previo y durante el embarazo, aumenta el riesgo para las dificultades de alimentación en la (el) niña(o), revistiendo importantes implicancias para la prevención y la intervención temprana de las dificultades alimentarias infantiles y futuras investigaciones en este campo $^{40,41}$. En virtud de lo anterior, van WezelMeijler y $\mathrm{Wit}^{29}$, advierten que con la creciente capacidad para inducir fertilidad en pacientes anorécticas, se deberían tomar seriamente en cuenta los efectos de un TCA materno en sus hijas(os).

Aunque von Soest y Wichstrøm ${ }^{42}$, señalan que la maternidad ejerce en general, un efecto positivo en los TCA, la lactancia representa un período crítico de adaptación para las madres con TCA por la combinación de los temores que conlleva la maternidad, los cambios en la silueta corporal y agravados por las variaciones de peso durante el embarazo, pudiendo exacerbarse su sintomatología y dar lugar a una depresión postparto que, 
más aun, involucra la aparición de actitudes irresponsables de rechazo a la hija(o), con la emergencia de complicaciones médicas y/o psicopatológicas que conllevan inclusive un riesgo vital, tanto para la paciente como para la (el) lactante l3-45. $^{4}$.

Según Micali y cols. ${ }^{46}$, los TCA maternos afectan la alimentación infantil y por ende el crecimiento y desarrollo desde el primer año de vida, por lo que los profesionales de la salud, ya sean pediatras, gineco-obstetras, médicos de familia, de atención primaria y otros miembros del equipo sanitario que se encuentran en contacto más estrecho con este tipo de pacientes, deberían permanecer alertas a estas manifestaciones para realizar una pesquisa diagnóstica precoz y poder manejarlas con un efectivo establecimiento de la alianza terapéutica, con énfasis en la psicoeducación, abordaje familiar y perentoria derivación a un equipo multidisciplinario en el cual ciertamente el especialista psiquiatra posee un rol relevante.

\section{Conclusiones}

Los TCA son desórdenes psicopatológicos multi-causales en cuya etiopatogenia emerge un reconocido influjo ambiental, en el cual cobra especial relevancia el contexto de desarrollo desde la infancia temprana. En este sentido, la impronta que establece un vínculo madre-hija (o) adquiere una relevancia trascendental, siendo muchas veces determinante en la eventual aparición de una patología alimentaria. Más aun, cuando la madre padece un trastorno de este tipo, la amenaza de provocar conductas alimentarias anormales e inclusive otro tipo de afecciones orgánicas y/o mentales en su progenie se torna todavía más patente. Es necesario por tanto acentuar la necesidad de su detección prematura, permitiendo enfocar una mejor aproximación terapéutica, preconizando una investigación permanente en la temática.

Potenciales conflictos de interés: Este trabajo cumple con los requisitos sobre consentimiento/asentimiento informado, comité de ética, financiamiento, estudios animales y sobre la ausencia de conflictos de intereses según corresponda.

\section{Referencias}

1.- Park RJ, Senior R, Stein A: The offspring of mothers with eating disorders. Eur Child Adolesc Psychiatry. 2003; 12: I110-9.

2.- Cordella P, Castro L, Díaz C, Zavala C, Lizana P: Las madres de adolescentes y jóvenes chilenos con trastornos alimentarios. Rev Med Chile 2009; 137: 785-90.

3.- American Psychiatric Association: Diagnostic and statistical manual of mental disorders. $3^{\text {rd }}$ Edition Revised. Washington, DC: American Psychiatric Association, 1987.

4.- American Psychiatric Association: Diagnostic and statistical manual of mental disorders. Text Revised. $4^{\text {th }}$ Edition. Washington, DC: American Psychiatric Association, 2000.

5.- Koubaa S, Hällström T, Hirschberg AL: Early maternal adjustment in women with eating disorders. Int $\mathrm{J}$ Eat Disord 2008; 41 (5): 405-10.

6.- Watkins B, Cooper PJ, Lask B: History of eating disorder in mothers of children with early onset eating disorder or disturbance. Eur Eat Disord Rev 2012; 20 (2): 121-5.

7.- Stein A, Woolley H, Cooper S, et al: Eating habits and attitudes among 10-year-old children of mothers with eating disorders: longitudinal study. Br J Psychiatry 2006; 189: 324-9.

8.- Field AE, Javaras KM, Aneja P, et al: Family, peer, and media predictors of becoming eating disordered. Arch Pediatr Adolesc Med 2008; 162 (6): 574-9.

9.- Farrow CV, Blissett J: Do obsessive compulsive symptoms mediate the relationship between maternal eating psychopathology and restrictive feeding practices? Int J Eat Disord 2009;42 (1): 76-80.

10.- Hoffman ER, Bentley ME, Hamer RM, et al: A comparison of infant and toddler feeding practices of mothers with and without histories of eating disorders. Matern Child Nutr 2012. doi: 10.1111/j.17408709.2012.00429.x.

11.- García de Amusquibar AM, De Simone CJ: Some features of mothers of patients with eating disorders. Eat Weight Disord 2003; 8 (3): 225-30.

12.- Powell FC, Farrow CV, Meyer C: Food avoidance in children. The influence of maternal feeding practices and behaviours. Appetite 2011; 57 (3): 683-92.

13.- Faith MS, Storey M, Kral TV, et al: The feeding de- 
mands questionnaire: assessment of parental demand cognitions concerning parent-child feeding relations. J Am Diet Assoc 2008; 108 (4): 624-30.

14.- Knoph B, Bulik CM, Von Holle A, et al: Psychosocial factors associated with broadly defined bulimia nervosa during early pregnancy: findings from the Norwegian mother and child cohort study. Aust N Z J Psychiatry. 2008; 42 (5): 396-404.

15.- Ammaniti M, Ambruzzi AM, Lucarelli L, et al: Malnutrition and dysfunctional mother-child feeding interactions: clinical assessment and research implications. J Am Coll Nutr 2004; 23 (3): 259-71.

16.- Cooper PJ, Whelan E, Woolgar M, et al: Association between childhood feeding problems and maternal eating disorder: role of the family environment. $\mathrm{Br} \mathrm{J}$ Psychiatry 2004; 184: 210-5.

17.- van den Berg PA, Keery H, Eisenberg M, et al: Maternal and adolescent report of mothers' weight-related concerns and behaviors: longitudinal associations with adolescent body dissatisfaction and weight control practices. J Pediatr Psychol 2010; 35 (10): 1093-102.

18.- Cooley E, Toray T, Wang MC, et al: Maternal effects on daughters' eating pathology and body image. Eat Behav 2008; 9 (1): 52-61.

19.- McCabe MP, Ricciardelli LA, Stanford J, et al: Where is all the pressure coming from? Messages from mothers and teachers about preschool children's appearance diet and exercise. Eur Eat Disord Rev 2007; 15 (3): 221-30.

20.- Gonçalves S, Silva M, Gomes AR, et al: Disordered eating among preadolescent boys and girls: the relationship with child and maternal variables. Nutrients. 2012; 4 (4): 273-85.

21.- Byely L, Archibald AB, Graber J, et al: A prospective study of familial and social influences on girls' body image and dieting. Int J Eat Disord 2000; 28 (2): 15564.

22.- Behar R: Trastornos de la conducta alimentaria: Clínica y epidemiología. En: Behar R, Figueroa G. ed. Trastornos de la conducta alimentaria. Segunda Edición, Santiago de Chile: Editorial Mediterráneo, 2010; 121-47.

23.- Rørtveit K, Aström S, Severinsson E: The meaning of guilt and shame: a qualitative study of mothers who suffer from eating difficulties. Int J Ment Health Nurs. 2010; 19 (4): 231-9.

24.- Rørtveit K, Aström S, Severinsson E: Experiences of guilt as a mother in the context of eating difficulties. Issues Ment Health Nurs 2009; 30 (10): 603-10.

25.- Bowlby J: Maternal care and mental health. $2^{\text {nd }}$ ed. Northvale, NJ; London: Jason Aronson, 1995.

26.- Behar R: Perspectiva evolucionista de los trastornos de la conducta alimentaria. Rev Med Chile 2012; 140: 513-9.

27.- Stein A, Woolley H, Senior R, et al: Treating disturbances in the relationship between mothers with bulimic eating disorders and their infants: a randomized, controlled trial of video feedback. Am J Psychiatry 2006; 163 (5): 899-906.

28.- Elfhag K, Linné Y: Gender differences in associations of eating pathology between mothers and their adolescent offspring. Obes Res 2005; 13 (6): 1070-6.

29.- van Wezel-Meijler G, Wit JM: The offspring of mothers with anorexia nervosa: a high-risk group for undernutrition and stunting? Eur J Pediatr 1989; 149 (2): 130-5.

30.- Russell GF, Treasure J, Eisler I: Mothers with anorexia nervosa who underfeed their children: their recognition and management. Psychol Med 1998; 28 (1): 93-108.

31.- Pike KM, Rodin J: Mothers, daughters, and disordered eating. J Abnorm Psychol 1991; 100 (2): 198-204.

32.- Tierney $S$, Fox JR, Butterfield $C$, et al: Treading the tightrope between motherhood and an eating disorder: a qualitative study. Int J Nurs Stud 2011t; 48 (10): 122333.

33.- Torgersen L, Ystrom E, Haugen M, et al: Breastfeeding practice in mothers with eating disorders. Matern Child Nutr 2010; 6 (3): 243-52.

34.- Waugh E, Bulik CM: Offspring of women with eating disorders. Int J Eat Disord 1999r; 25 (2): 123-33.

35.- Agras S, Hammer L, McNicholas F: A prospective study of the influence of eating-disordered mothers on their children. Int J Eat Disord 1999; 25 (3): 253-62.

36.- Stapleton H, Fielder A, Kirkham M: Breast or bottle? Eating disordered childbearing women and infant-feeding decisions. Matern Child Nutr 2008; 4 (2): 106-20.

37.- Rodgers $R$, Chabrol H: Parental attitudes, body image disturbance and disordered eating amongst adolescents and young adults: a review. Eur Eat Disord Rev 2009; 17 (2): 137-51.

38.- Blissett J, Haycraft E: Parental eating disorder symptoms and observations of mealtime interactions with children. J Psychosom Res 2011; 70 (4): 368-71.

39.- Garner DM: Eating disorders inventory-2: Professional manual. Odessa, FL, Psychological Assessment Resources, 1991.

40.- Carwell ML, Spatz DL: Eating disorders \& breastfeeding. J Child Psychol Psychiatry 2011; 52 (7): 800-7.

41.- Vignalou J, Guedeney N: About offsprings of mothers with anorexia nervosa: what about clinical practice in pediatric settings?. Arch Pediatr 2006; 13 (5): 484-7.

42.- von Soest T, Wichstrøm L: The impact of becoming a mother on eating problems. Int J Eat Disord 2008; 41 
(3): $215-23$

43.- Abraham S, Taylor A, Conti J: Postnatal depression, eating, exercise, and vomiting before and during pregnancy. Int J Eat Disord 2001; 29 (4): 482-7.

44.- Astrachan-Fletcher E, Veldhuis $C$, Lively $N$, et al: The reciprocal effects of eating disorders and the postpartum period: a review of the literature and recommendations for clinical care. J Womens Health (Larchmt) 2008; 17
(2): 227-39.

45.- Behar R, González J, Ariza M, et al: Trastornos de la conducta alimentaria en mujeres embarazadas controladas en atención primaria. Rev Chil Obstet Ginecol 2008; 73 (3): 155-62.

46.- Micali N, Simonoff E, Treasure J: Infant feeding and weight in the first year of life in babies of women with eating disorders. J Pediatr 2009; 154 (1): 55-60. 